\title{
UMA AVALIAÇÃO DE IMPACTO DE UM PROGRAMA DE QUALIFICAÇÃO PROFISSIONAL NA EMPRESA SOBRE A INSERÇÃO DOS JOVENS NO MERCADO DE TRABALHO FORMAL
}

\author{
Carlos Henrique L. Corseuil * \\ Miguel Foguel ${ }^{+}$ \\ LEON FACEIRA TOMELIN $\ddagger$
}

\begin{abstract}
Resumo
Neste artigo, avaliamos o impacto de um programa de treinamento para jovens que ocorre integralmente dentro das empresas participantes. Utilizando métodos baseados em pareamento com escore de propensão e dados da Relação Anual de Informações Sociais (RAIS), investigamos a inserção laboral dos participantes do programa relativamente à dos jovens aprendizes, que formam um grupo de controle pertinente para medir efeitos diferenciados do programa. Os resultados indicam que o programa afeta positivamente a empregabilidade dos participantes, aumentando as chances de obter emprego formal e em empresas grandes. Já o impacto sobre salários foi nulo no curto e negativo no médio prazo.
\end{abstract}

Palavras-chave: treinamento; jovens; mercado de trabalho.

\begin{abstract}
In this paper, we evaluate the impact of a training program for youths that takes place entirely inside the participant firms. Employing methods based on propensity score matching and RAIS data, we investigate the labor market insertion of participants relatively to that of apprentices, who form a pertinent control group for measuring differentiated effects of the program in focus. Our results indicate that the program positively affects the employability of participants, increasing their chances of obtaining formal employment and employment in large firms. Regarding wages, the impacts were null in the short run and negative in the medium run.
\end{abstract}

Keywords: training; youth; labor market.

JEL classification: JEL: J13; J24; J38

DOI: http : / / dx.doi .org/10 .11606/1980-5330/ea141238

\footnotetext{
* Técnico de Planejamento na Diretoria de Estudos e Políticas Sociais (Disoc) do Ipea. E-mail: carlos.corseuil@ipea.gov.br.

† Técnico de Planejamento na Disoc/Ipea. E-mail: miguel.foguel@ipea.gov.br.

$\ddagger$ Pesquisador do Programa de Pesquisa para o Desenvolvimento Nacional (PNPD) na Disoc/Ipea. E-mail: leon.tomelin@ipea.gov.br.
} 


\section{Introdução}

A difícil inserção dos jovens no mercado de trabalho motiva análises em diversos países do mundo. Os volumes editados pela Organização para a Cooperação e Desenvolvimento Econômico - OECD (2014) e Freeman \& Wise (1982) - comprovam que essa preocupação já vem de longa data. Não só os acadêmicos se preocupam com o problema das altas taxas de desemprego dos jovens, mas também os gestores de política pública e diversas entidades da sociedade civil. Ao longo das últimas décadas, uma miríade de políticas ativas de mercado de trabalho especificamente voltada para o grupo de jovens foi criada em diversos países desenvolvidos e em desenvolvimento.

No Brasil, o estudo da inserção dos jovens no mercado de trabalho ganhou escopo nos últimos anos com uma proliferação de análises. Os volumes editados por OECD (2014) e Corseuil \& Botelho (2014)), por exemplo, são inteiramente dedicados a esse tema. Da mesma forma, que no contexto internacional, vários programas que pretendem melhorar a situação dos jovens no mercado de trabalho também foram criados no Brasil.

O principal objetivo deste estudo é avaliar o impacto de uma iniciativa pioneira de qualificação profissional para jovens sobre sua futura inserção no mercado de trabalho. Esse programa traz como elemento novo o fato de o treinamento ser ministrado integralmente dentro das dependências e por profissionais das empresas que fazem parte da iniciativa. O programa é concebido por uma instituição privada sem fins lucrativos, que também assessora as empresas no que tange à sua implementação. Devido a um acordo de confidencialidade estabelecido com essa instituição - que chamaremos de "instituição $\mathrm{Y}^{\prime \prime}$-, no restante do texto faremos referência a essa iniciativa de qualificação como "programa Q".

Algumas características do programa $Q$ tornam sua avaliação interessante. Primeiro, o desenvolvimento de atividades no interior das empresas permite que estas tenham a oportunidade de conhecer melhor o potencial produtivo do jovem, mitigando assim os problemas típicos de assimetria de informação na contratação de trabalhadores, especialmente de jovens, que não possuem um histórico longo no mercado de trabalho.

O segundo elemento interessante é que o programa utiliza uma metodologia de treinamento que contempla uma customização para cada empresa participante. ${ }^{1}$ Ou seja, o treinamento tende a ter um componente com teor mais específico do que a prática em programas similares. Essa possibilidade tende a ser reforçada pelo fato de o treinamento ser conduzido por funcionários da empresa que fornece o treinamento.

O impacto do programa em questão será avaliado em dimensões relacionadas à inserção dos jovens no mercado de trabalho formal em um período de até três anos após a conclusão da qualificação. Diversos estudos sobre inserção de jovens no mercado de trabalho sugerem um quadro no qual o jovem enfrenta dificuldades não apenas de acessar o emprego formal, mas também de se manter nele. Tanto Leighton \& Mincer (1982) como Clark \& Summers (1982) mostraram que as altas taxas de desemprego para jovens nos Estados Unidos são consequência de uma alta frequência no desemprego e não de uma duração prolongada nesse estado. Flori (2005) constata o mesmo para o Brasil nos anos 90, e Corseuil et al. (2014) mostram que, mesmo no setor formal, a

\footnotetext{
${ }^{1}$ A metodologia é desenvolvida pela instituição Y.
} 
rotatividade é extremamente alta para os jovens. Tanto a inserção do jovem no mundo do trabalho como a sua permanência aparecem explicitamente entre os objetivos do programa Q. Em linha com as evidências e com os objetivos do programa, procuramos incorporar essas duas dimensões neste estudo.

Outra dimensão incorporada na literatura sobre o tema diz respeito à qualidade do emprego em que o jovem eventualmente vem a se inserir. Há evidências (Gregg \& Tominey 2005, Eliason \& Storrie 2006, Cruces et al. 2012) de que uma inserção marcada por passagens em empregos precários e/ou períodos de desemprego tende a comprometer toda a trajetória profissional futura dos jovens. Dessa forma, aproveitaremos a riqueza das informações de que dispomos para incorporar também aspectos relacionados à qualidade dos empregos eventualmente obtidos pelos jovens após completarem sua qualificação profissional.

A base de dados que construímos para realizar a avaliação de impacto aqui proposta combina dois registros administrativos. A primeira fonte são dados fornecidos pela instituição responsável pelo programa. A segunda fonte é a Relação Anual de Informações Sociais (Rais) do Ministério do Trabalho (Brasil, 2009).

A Rais é um registro administrativo baseado em declarações anuais de todas as empresas do setor formal (com Cadastro Nacional de Pessoas Jurídicas - CNPJ) a respeito de todos os empregados com os quais estabeleceram vínculos trabalhistas. Destaca-se enquanto fonte de informação do mercado de trabalho, tanto em virtude de sua cobertura quanto de seu conteúdo. Em relação à cobertura não há qualquer tipo de restrição, frequente em outras bases, como por exemplo em relação a tamanho ou setor das empresas. Já no que diz respeito ao conteúdo, a Rais contempla em seus dados primários informações referentes: i) ao estabelecimento; ii) ao trabalhador; e iii) ao contrato de trabalho. No primeiro grupo de informações usamos o setor de atividade, a Unidade da Federação (UF) de localização, a natureza jurídica e o número de empregados (vínculos ativos) em 31/12 de cada ano. No segundo grupo utilizamos informações referentes à idade e à escolaridade do trabalhador. As informações referentes ao contrato de trabalho são os meses de admissão e desligamento, o tipo de contrato (prazo determinado ou indeterminado) e a remuneração.

Vale dizer que tanto os trabalhadores como os estabelecimentos (e as firmas) são identificados de forma única e coerente ao longo do tempo. Dessa forma, valemo-nos da informação longitudinal para construir a trajetória dos jovens após a qualificação. Mais detalhes sobre a construção do nosso banco de dados serão fornecidos na próxima seção.

Uma questão fundamental em toda avaliação de impacto é a escolha do contrafactual apropriado. O ideal seria contrastar os resultados dos jovens qualificados nas empresas com os seus resultados, caso não houvessem participado desse programa. Tal comparação não é factível no mundo real. Portanto, é necessário recorrer a resultados observados para outro grupo de trabalhadores que sejam parecidos com o grupo que passou pelo programa, ou seja, é preciso encontrar um grupo de controle.

Neste artigo, usamos as informações disponíveis na Rais para jovens aprendizes para formar o grupo de comparação. Essa escolha se justifica por dois motivos. Em primeiro lugar, a instituição Y não dispunha de dados referentes aos indivíduos não selecionados para o programa Q. Uma alternativa que nos parece apropriada é compará-los com os participantes de um programa que 
tenha algum componente de treinamento no emprego, que é o caso do programa de aprendizagem. A escolha dos aprendizes como grupo de controle nos permite homogeneizar os jovens dos dois grupos no que diz respeito a uma dimensão tipicamente não observada por avaliadores, a saber, a motivação por fazer algum curso de treinamento. Em segundo lugar, a aprendizagem é tida como a melhor prática para promover a inserção de jovens no mercado de trabalho com qualidade (Biavaschi et al. 2013).

Admitindo que nossa amostra é suficientemente homogênea em relação à dimensão não observável da motivação para realizar cursos de treinamento, nos sentimos mais confiantes em empregar métodos de avaliação baseados somente em características observáveis. Nossa confiança também é respaldada pela riqueza de características observáveis que podemos utilizar a partir dos dados da Rais. Os métodos que utilizamos foram de dois tipos: i) pareamento com escore de propensão; e ii) regressão linear. No primeiro caso seguimos as recomendações de Huber et al. (2013) e utilizamos o critério do raio para a definição dos contrafactuais para os indivíduos tratados. No segundo caso, seguimos as recomendações de Kline (2011) e combinamos modelos de regressão lineares com a decomposição de Oaxaca-Blinder para obter uma estimação duplamente robusta. Mais detalhes sobre a metodologia serão apresentados na seção 4. Antes disso provemos um pouco mais de informações sobre o programa $\mathrm{Q}$ e o comparamos com outras iniciativas de treinamento para jovens na seção 2. A seção 3 detalha a construção da nossa base de dados, bem como apresenta algumas características básicas dos jovens que compõem nossa amostra. Nossos resultados principais são apresentados na seção 5.

\section{Contexto}

A dificuldade de inserção do jovem no mercado de trabalho fez proliferarem no mundo diversas iniciativas de qualificação para jovens. Os impactos dessas iniciativas sobre sua inserção laboral têm sido investigados por uma extensa lista de estudos de avaliação para vários países. Baseados em um arcabouço meta-analítico, Card et al. (2010) e Kluve (2010) resumem suas constatações a respeito da avaliação de diversas Políticas Ativas de Mercado de Trabalho (PAMTs) para um conjunto amplo de países. Ambos os estudos concluem que programas de treinamento voltados para jovens são menos bem-sucedidos que outros tipos de PAMTs.

Esse quadro fez com que novas alternativas para além do formato tradicional de treinamento de conteúdo específico em sala de aula fossem consideradas. Novos formatos vieram com diferentes propostas, de certa forma complementares. Em alguns casos optou-se por abrir o leque no conteúdo, de forma a incluir não apenas o conhecimento necessário para desempenhar um único ofício. Exemplos desse formato são programas que alargam o escopo das iniciativas tradicionais para inserir um conteúdo de habilidades socioemocionais. Um programa desse tipo é oferecido pela Organização Não Governamental (ONG) Galpão Aplauso no Rio de Janeiro, e foi objeto de uma avaliação de impacto conduzida por Calero et al. (2017). A proposta é formar uma qualificação mais abrangente, cuja motivação vai além da empregabilidade imediata do jovem.

Em outros casos, optou-se por contemplar o jovem com um contato mais próximo com as empresas durante seu treinamento, combinando parte do pe- 
ríodo em sala de aula e parte do período com atividades práticas nas empresas. Talvez o melhor exemplo de iniciativa desse tipo no Brasil seja o contrato de aprendizagem, que foi objeto de avaliação por Corseuil et al. (2015).

\subsection{A intervenção a ser avaliada}

O programa $Q$ se insere na segunda linha de programas de treinamento exposta acima, que tende a aproximar mais o jovem em treinamento com o mundo corporativo. Na realidade, a proposta do programa é ir ao limite dessa aproximação, fazendo toda a qualificação dos jovens dentro da empresa, inclusive com funcionários da empresa atuando como instrutores tanto na parte teórica como na prática. Especificamente, o programa Q oferece cursos de educação profissional dentro de empresas de médio e grande porte. Além de oferecer as instalações e equipamentos, as empresas disponibilizam o tempo e o talento acumulado pelos seus empregados, que atuam de forma voluntária como educadores dos cursos. Estes são confeccionados em conformidade com as características do negócio de cada empresa, que procura levar em consideração os aspectos da comunidade e do mercado de trabalho da localidade onde estão inseridas. A parte teórica dos cursos devem procurar desenvolver tanto as competências pessoais quanto as habilidades profissionais do público-alvo do programa, que é formado por jovens cursando o ensino médio noturno e provenientes de famílias de baixa renda. Cada curso na empresa forma 20 jovens anualmente, tem carga horária mínima de 800 horas-aula e recebe certificação, avalizada pelo Ministério da Educação, de instituições federais de ensino superior conveniadas com a instituição Y. A carga horária do curso é dividida em $85 \%$ para a parte teórica e $15 \%$ para a parte prática. Além do curso na empresa, o jovem participante recebe bolsa auxílio de meio salário mínimo, alimentação, transporte, uniforme, assistência médica e seguro de vida.

O programa Q pode ser visto como parte de um conjunto de iniciativas conhecido como "ação voluntária empresarial" . De acordo com levantamento elaborado pela Fundação Itaú Social (2015) não há avaliações de impacto disponíveis para esse tipo de iniciativa. Dessa forma, esse é o primeiro estudo de avaliação de impacto de um programa de treinamento fundamentado nessa prática.

Há na literatura alguns resultados de análises de programas de treinamento baseados puramente em componentes do tipo on the job training. Biavaschi et al. (2013) sintetizam uma vasta literatura e apontam que esse tipo de programa pode levar a bons resultados no curto prazo. Porém, os autores alertam que a especificidade do conhecimento acumulado nesse tipo de treinamento tende a levar os jovens a uma posição de maior vulnerabilidade no médio prazo em virtude de uma menor valorização desse conhecimento por outros potenciais empregadores.

\subsection{O grupo de comparação}

Nesta subseção, analisamos as principais semelhanças e diferenças entre o programa Q e o do jovem aprendiz, cujos participantes formam o grupo de comparação utilizado para a avaliação de impacto do primeiro programa.

Um primeiro aspecto em comum a ressaltar é que ambos os programas são de treinamento profissional, o que implica que, pelo menos potencialmente, a 
decisão de participar neles deve estar associada a preferências e características não observáveis (pelo analista) dos jovens tais como motivação, curiosidade e conscienciosidade. A existência desse tipo de semelhança entre os jovens dos dois programas é importante pois os torna mais homogêneos em dimensões de difícil mensuração antes dos programas, reduzindo assim a possibilidade de viés de seleção na identificação dos efeitos de interesse.

Outra semelhança importante a se destacar é que, tal como no programa Q, os aprendizes também têm uma formação dividida em uma parte teórica e outra prática. A carga horária da parte teórica na aprendizagem pode variar entre 400 e 800 horas, ao passo que no programa Q é estipulada em 680 horas ( $85 \%$ de 800 horas). Outra dimensão comum é que na aprendizagem a formação teórica também se inicia pelas competências pessoais antes de contemplar competências voltadas a atividades práticas desenvolvidas no mercado de trabalho. No aspecto remuneratório, grande parte dos aprendizes ganha um salário mínimo, o que deve ser valorado pelo jovem de forma semelhante ao pacote oferecido pelo programa $Q$ (1/2 salário mínimo + benefícios). Por fim, a formação teórica na aprendizagem também é chancelada pelo governo federal.

Em suma, para jovens que anseiam por uma experiência prática no mercado de trabalho - e, portanto, veem como um atrativo não só a remuneração mas também a parte prática da formação - e estão dispostos a devotar uma carga semelhante do treinamento à parte teórica, as características dos dois programas parecem ser suficientemente próximas para torná-los equivalentemente atrativos.

No entanto, apesar dessas semelhanças, há diferenças em algumas dimensões dos dois programas. Em primeiro lugar, a parte teórica da aprendizagem é fornecida em turmas que agregam jovens contratados por diferentes empresas. Dessa forma, há um limite para o grau de especificidade desse conteúdo, em contraponto ao programa $\mathrm{Q}$, no qual a parte teórica é oferecida em turmas específicas para cada uma das empresas participantes possibilitando o desenho customizado do conteúdo a ser passado para os alunos. Em segundo lugar, a carga total de treinamento tende a ser maior para o aprendiz. As 800 horas que definem a carga máxima do programa $Q$ tendem a ser menores que no programa de aprendizes, cujo total varia entre 800 e 1600 horas. Dada a semelhança na carga horária da parte teórica, deduz-se que a carga horária na parte prática é mais extensa na aprendizagem. Essas diferenças entre os tipos de treinamento providos pelas respectivas intervenções facilitam a interpretação de eventuais resultados que apontem para efeitos diferenciados entre os dois programas.

\section{Preliminares Empíricos}

\subsection{Construção da base de dados}

A partir dos dados da Rais e de informações fornecidas pela instituição Y foi possível construir uma base de dados contendo informações a respeito dos indivíduos que foram qualificados pelo programa $\mathrm{Q}$ ou pela aprendizagem. Além de características individuais, foi possível reunir informações referentes às respectivas empresas onde o jovem exerceu a dimensão prática do seu treinamento (seja na condição de aprendiz ou de aluno do programa Q), bem como informações referentes a um eventual emprego obtido após esse treina- 
mento. Para se chegar em uma base com todas essas informações foi necessário percorrer três etapas.

Tabela 1: Origem da informação para jovens do programa Q

\begin{tabular}{l|c|c}
\hline & $\begin{array}{c}\text { Cadastro da } \\
\text { instituição Y }\end{array}$ & RAIS \\
\hline Identificação do indivíduo & $\mathrm{X}$ & $\mathrm{X}$ \\
Ano de conclusão do treinamento & $\mathrm{X}$ & \\
Educação & $\mathrm{X}$ & \\
Idade & $\mathrm{X}$ & \\
UF & & $\mathrm{X}$ \\
Indicador de experiência prévia no setor formal & & $\mathrm{X}$ \\
ID da empresa onde fez treinamento prático & & $\mathrm{X}$ \\
Tamanho da empresa onde fez treinamento & & $\mathrm{X}$ \\
Setor de atividade dessa empresa & & $\mathrm{X}$ \\
Indicador de emprego pós treinamento & & $\mathrm{X}$ \\
ID da empresa empregadora pós treinamento & & $\mathrm{X}$ \\
Tamanho da empresa pós treinamento & & $\mathrm{X}$ \\
Salário médio da empresa pós treinamento & & $\mathrm{X}$ \\
Tipo de contrato do jovem nessa empresa & & $\mathrm{X}$ \\
Salário do jovem nessa empresa & &
\end{tabular}

Fonte: Instituição Y e Rais.

Elaboração dos autores.

Em uma primeira etapa, complementamos as informações dos jovens qualificados pelo programa Q provenientes da instituição Y com informações vindas da Rais, tal como descrito na Tabela 1. Nosso ponto de partida corresponde às informações de jovens que concluíram o treinamento do programa Q entre os anos de 2006 e $2012 .^{2}$ Nessa etapa, de um total de 5.657 jovens da base de dados do programa $Q$, tivemos que descartar 587 por não os encontrar em nenhuma empresa com informação disponível na Rais no respectivo ano de conclusão do treinamento.

Em uma segunda etapa, buscamos essas mesmas informações para jovens que estavam concluindo algum contrato de aprendizagem nos mesmos anos de conclusão dos jovens treinados pelo programa Q. A diferença do ponto de vista operacional é que no caso dos aprendizes todas as informações são provenientes da Rais.

Finalmente, na terceira e última etapa reunimos as informações das duas etapas anteriores em uma única base e aplicamos alguns filtros adicionais com o intuito de aumentar a homogeneidade entre os dois grupos de jovens. Os filtros aplicados consistem em manter na base somente as informações que atendessem as seguintes condições:

\footnotetext{
${ }^{2} \mathrm{O}$ arquivo que nos foi enviado compreendia informações para jovens que concluíram o programa Q entre 2004 e 2013. Excluímos os anos de 2004 e 2005 por dois motivos: i) por ter havido uma mudança no critério de elegibilidade para a aprendizagem em 2005; e, ii) por ter relativamente poucos jovens concluindo o programa Q nesses anos. Já o ano de 2013 foi excluído porque é também o último ano para o qual dispomos de informações da Rais. Como temos interesse em informações relativas a um eventual emprego ao menos um ano após o treinamento, torna-se necessário limitar a amostra dessa forma.
} 
- a idade do jovem no momento de conclusão do treinamento deve ser inferior a 24 anos;

- a duração do vínculo dos aprendizes deve estar contida no intervalo entre 10 e 12 meses;

- os jovens devem ter pelo menos o ensino fundamental completo;

- a empresa onde foi realizado o treinamento deve ter ao menos 200 empregados;

- a empresa onde foi realizado o treinamento deve estar situada no Rio Grande do Sul, em São Paulo ou em Minas Gerais; e

- a empresa onde foi realizado o treinamento deve ser privada, e não agrícola.

Essas restrições à amostra foram implementadas devido às características dos jovens e das empresas participantes do programa Q.

Ao final da terceira etapa, chegamos a um banco de dados com informações para 7.235 jovens, dos quais 2.463 foram treinados no programa $Q$, e 4.772, pela aprendizagem. As Tabelas 2 e 3 nos indicam as semelhanças entre esses dois grupos de jovens que compõem a nossa base.

A Tabela 2 reporta as frequências relativas dos jovens de acordo com características dos indivíduos. Pode-se constatar que, apesar dos filtros aplicados, algumas diferenças importantes permanecem em nossa amostra. A diferença mais marcante aparece quando comparamos as frequências relativas por faixa de escolaridade. Enquanto apenas 13,8\% dos jovens de nossa amostra final provenientes da aprendizagem possuem o ensino médio completo, $45,2 \%$ atendem essa condição entre os jovens provenientes do programa Q.

As diferenças por faixa etária também são nítidas, embora em menor intensidade do que as reportadas para escolaridade: $56,0 \%$ dos jovens provenientes do programa Q são menores de idade, ao passo que essa proporção é de $45,8 \%$ para os jovens provenientes da aprendizagem. Por fim, vale registrar que, no que diz respeito à experiência prévia de emprego em empresas registradas na Rais, a frequência relativa é bem similar nos dois grupos $(7,1 \%$ e 6,9\%).

Tabela 2: Frequência relativa dos jovens de acordo com características individuais

\begin{tabular}{lcc}
\hline & Aprendizagem & Programa Q \\
\hline Escolaridade & & \\
$\quad$ Fundamental completo & 85,8 & 54,6 \\
Médio completo & 13,8 & 45,2 \\
Superior completo & 0,4 & 0,2 \\
& & \\
Idade & 45,8 & 56,0 \\
14 a 17 anos & 54,2 & 44,0 \\
18 a 23 anos & 7,1 & 6,9 \\
Experiência Prévia & & \\
\hline Fonte: Rais. & & \\
Elaboração dos autores. &
\end{tabular}

A Tabela 3 traz uma comparação análoga, porém, com frequências relativas a características da empresa onde o jovem se qualificou. As diferenças 
entre jovens provenientes da aprendizagem e do programa $\mathrm{Q}$ tendem a ser ainda mais acentuadas nas dimensões reportadas nessa tabela. Por exemplo, no que diz respeito ao setor de atividade da empresa onde o jovem se qualificou, a indústria responde por $89,0 \%$ entre aqueles provenientes do programa $\mathrm{Q}$, e por apenas $32,2 \%$ entre os oriundos da aprendizagem. Outra diferença marcante aparece na análise por porte da empresa, quando medido em número de empregados. O maior porte (mais de 500 empregados) responde por $76,8 \%$ dos casos nos jovens vindos do programa Q, e por $51,9 \%$ nos oriundos da aprendizagem. A última dimensão avaliada foi relativa à localização geográfica, onde se vê uma concentração de jovens no Estado de São Paulo, especialmente para os jovens provenientes do programa $\mathrm{Q}(74,7 \%)$.

Em resumo, as tabelas demonstram que os filtros aplicados à amostra inicial não são suficientes para eliminar as diferenças entre o grupo de jovens qualificados pela aprendizagem e o grupo de jovens qualificados pelo programa Q. As maiores diferenças estão associadas com uma maior concentração dos jovens do segundo grupo na faixa de educação mais elevada e em empresas de maior porte e da indústria. Note que todas essas características mais presentes entre os jovens qualificados pelo programa $Q$ estão associadas a maiores salários e melhores perspectivas de emprego. Logo, é preciso levar em conta tal diferença ao estimar o efeito desse programa. Como veremos mais adiante, se essas dimensões forem suficientes para captar as diferenças remanescentes entre os dois grupos, o método de seleção em observáveis aqui empregado será capaz de isolar o efeito do programa. Isso, no entanto, não será verdadeiro se existirem fatores não observáveis que influenciam a entrada em cada um dos programas e também afetam a empregabilidade e o salário futuro do jovem.

Tabela 3: Frequência relativa dos jovens de acordo com características das empresas

\begin{tabular}{lcc}
\hline & Aprendizagem & Programa Q \\
\hline Setor (treinamento) & 32,2 & \\
$\quad$ Indústria & 3,1 & 89,0 \\
$\quad$ Construção & 14,6 & - \\
$\quad$ Comércio & 50,0 & 11,0 \\
$\quad$ Serviços & & \\
Tamanho da Empresa (treinamento) & 48,1 & 23,2 \\
$\quad$ De 200 a 500 empregados & 51,9 & 76,8 \\
De 500 ou mais empregados & & \\
Região/UF & 43,9 & 16,3 \\
MG & 48,1 & 74,7 \\
SP & 8,0 & 9,0 \\
$\quad$ RS & & \\
\hline Fonte: Rais. & & \\
Elaboração dos autores. & & \\
&
\end{tabular}

\subsection{Dimensões a serem avaliadas}

Em muitos casos, os programas de qualificação de jovens são avaliados em uma única dimensão: a obtenção ou não de qualquer tipo de emprego em uma curto espaço de tempo após a qualificação. Essa certamente é uma dimensão importante de ser avaliada, e será considerada neste estudo. No entanto, conforme mencionado na introdução, entendemos ser importante avaliar o im- 
pacto do programa de qualificação para além desse resultado mais direto na dimensão de empregabilidade. Assim, ampliamos o escopo da avaliação em duas direções. Primeiro, ampliamos o leque de variáveis a serem avaliadas para que seja possível captar também o impacto sobre outras dimensões do emprego eventualmente obtido. Nesse sentido, analisaremos também a probabilidade de se empregar: com contrato formal por tempo indeterminado, em empresas grandes (com mais de 200 empregados) e em empresas que pagam salários relativamente altos. A justificativa para essas variáveis é que tanto o contrato por tempo indeterminado como o emprego em empresas maiores, e que pagam mais, são indicadores da qualidade e da produtividade do posto de trabalho a que o participante do programa $Q$ teve acesso.

Além disso, consideramos dois horizontes distintos para avaliar tanto a empregabilidade como a dimensão qualitativa do emprego. Um horizonte compreende o ano de conclusão do treinamento e o ano seguinte (que denominaremos curto prazo); o outro compreende os dois anos subsequentes, ou seja, do segundo ano após a conclusão do treinamento até o terceiro (médio prazo).

A Tabela 4 apresenta médias de algumas variáveis de resultado para os dois grupos utilizados na análise: os participantes do programa Q e os aprendizes. No primeiro par de colunas, reportamos indicadores de empregabilidade e salário para o curto prazo, e no segundo reportamos os mesmos indicadores para o médio prazo. A primeira linha indica que, embora a chance de obter emprego formal seja distinta entre os dois horizontes temporais, ela é parecida entre os dois grupos de análise. As diferenças entre os grupos deixam de ser desprezíveis nos indicadores reportados nas demais linhas da tabela. No curto prazo, a proporção de jovens qualificados pelo programa Q que permanecem na mesma empresa onde realizaram o treinamento é de $25,5 \%$ , ao passo que essa proporção é de somente $16,2 \%$ entre aprendizes. A diferença nesse indicador aumenta quando analisamos os resultados para o médio prazo, com $28,0 \%$ e 13,0 \% dos participantes do programa Q e dos aprendizes permanecendo na mesma empresa, respectivamente.

Há diferenças também no curto prazo entre percentuais de jovens dos dois grupos que conseguem um emprego formal regido por contrato por tempo indeterminado, com vantagem para os aprendizes $(68,9 \%)$ em relação aos jovens do programa $Q(60,8 \%)$. É interessante notar que no médio prazo essa diferença torna-se pequena e até se inverte, já que os participantes do programa Q experimentam um aumento da probabilidade de estarem empregados por prazo indeterminado maior que os aprendizes entre o curto e o médio prazos.

A Tabela 4 também reporta a remuneração média entre os jovens que se empregam no setor formal após o treinamento. ${ }^{3}$ A quarta linha indica que a média salarial é maior para os jovens provenientes do programa $\mathrm{Q}$ em qualquer um dos dois horizontes analisados. Observa-se uma média de $\mathrm{R} \$ 647,48$ para os jovens do programa $\mathrm{Q}$ empregados no horizonte mais próximo à conclusão da qualificação, e de $\mathrm{R} \$ 560,09$ para os jovens oriundos da aprendizagem que conseguem emprego nesse mesmo horizonte. ${ }^{4}$ Ao considerar o horizonte de médio prazo, os valores sobem para $\mathrm{R} \$ 854,48$ e $\mathrm{R} \$ 848,06$, respectivamente. Na última linha, reportamos resultados análogos para remuneração

\footnotetext{
${ }^{3}$ Como pode haver variação do ano em que o salário é observado, deflacionamos os valores expressando todos os salários em reais de 2013.

${ }^{4}$ Em caso de múltiplos empregos para um jovem dentro de um dos horizontes considerados, computamos a média dos salários recebidos por esse jovem.
} 
Tabela 4: Indicadores de empregabilidade e remuneração obtidos após o treinamento

\begin{tabular}{lc|c|c|c}
\hline & \multicolumn{2}{c|}{$\begin{array}{c}\text { Ano do treinamento } \\
\text { ou no seguinte }\end{array}$} & \multicolumn{2}{c}{$\begin{array}{c}\text { Dois ou três anos } \\
\text { depois do treinamento }\end{array}$} \\
\cline { 2 - 5 } & Aprendizagem & Programa Q & Aprendizagem & Programa Q \\
\hline $\begin{array}{l}\text { Emprego } \\
\begin{array}{l}\text { Emprego na } \\
\text { mesma empresa }\end{array}\end{array}$ & 71,5 & 69,1 & 88,8 & 90,2 \\
$\begin{array}{l}\text { Contratado por } \\
\text { tempo indeterminado }\end{array}$ & 68,9 & 25,5 & 13,0 & 28,0 \\
$\begin{array}{l}\text { Remuneração mensal } \\
\text { pós-treinamento }\end{array}$ & 560,09 & 60,8 & 86,0 & 87,1 \\
$\begin{array}{l}\text { Remuneração horária } \\
\text { pós-treinamento }\end{array}$ & 13,75 & 15,83 & 848,06 & 854,48 \\
\hline $\begin{array}{l}\text { Fonte: Rais. } \\
\text { Elaboração dos autores. }\end{array}$ & & & & 21,32 \\
\end{tabular}

padronizada por horas trabalhadas na semana. Do ponto de vista qualitativo, mantém-se a vantagem dos jovens oriundos do programa $\mathrm{Q}$ em ambos os horizontes.

\section{Metodologia}

\subsection{Aspectos introdutórios}

A avaliação dos efeitos de um programa é tipicamente realizada com base na busca de um grupo de controle que deve representar o que ocorreria com o grupo de tratamento caso este não tivesse participado do programa. Idealmente, para que o grupo de controle represente bem essa situação contrafactual é importante que ele seja semelhante, pelo menos em média, ao grupo tratado tanto nas características observáveis pelo analista (por exemplo, idade e educação) quanto nas não observáveis (por exemplo, motivação, habilidades cognitivas e não cognitivas).

Entretanto, nem sempre é fácil construir um grupo de controle que atenda a essas exigências. De fato, embora o método baseado na seleção aleatória dos participantes dos dois grupos seja capaz de gerar esse tipo de grupo de controle, normalmente as avaliações são implementadas por meio de métodos mais restritivos que lançam mão de hipóteses para contornar essa dificuldade. Neste estudo, empregaremos dois métodos cuja hipótese fundamental é que as características observáveis dos indivíduos antes do programa são suficientes para tornar os dois grupos semelhantes.

A riqueza de informações dos dados disponíveis é um dos principais determinantes para a adequação da hipótese acima. No nosso caso, a hipótese se mostrará adequada na medida em que tivermos informações sobre características dos jovens que afetam sua empregabilidade e a participação em cada programa. Dentre as informações disponíveis na base de dados, utilizamos a escolaridade, a faixa etária, se tinha experiência prévia no setor formal, o setor de atividade da empresa onde se qualificou, a UF onde estava localizada essa empresa e o ano de conclusão da qualificação. Não é possível afirmar que o uso desse conjunto de variáveis seja suficiente para tornar os dois grupos de jovens homogêneos. Em particular, uma vez que o programa Q é voltado para jovens de baixa renda, pode-se argumentar que nesse conjunto de variáveis está ausente a renda domiciliar per capita dos jovens no início do treinamento ou a escolaridade de seus pais. Essas variáveis tendem a captar dimensões im- 
portantes sobre a qualidade do capital humano, acumulado pelo jovem, que podem simultaneamente afetar a participação nos programas e a inserção no mercado de trabalho (formal). Infelizmente, no entanto, não dispomos dessas variáveis para os aprendizes, já que a Rais não contém informações sobre os domicílios dos trabalhadores.

Tendo essa limitação dos dados em conta, empregamos dois métodos baseados na hipótese de seleção em observáveis. Especificamente, estimaremos os impactos do programa $\mathrm{Q}$ com base no método semiparamétrico de pareamento com escore de propensão e o método paramétrico de regressão linear baseado nas ideias da decomposição de Oaxaca-Blinder. A escolha do primeiro método segue recomendação de Huber et al. (2013), que comparam diversas alternativas em um contexto de avaliação de programas de emprego, bastante similar ao nosso. Já o segundo método se mostra bem adequado a contextos no quais o número de observações no grupo de controle é bem maior do que no grupo de tratados, tal como no nosso caso. Além disso o desempenho desse método também se mostrou satisfatório na análise de Huber et al. (2013).

Ambos os métodos procuram estimar um mesmo parâmetro que pode ser derivado do seguinte arcabouço conceitual. Considere uma amostra de $i=$ $1, \ldots, N$ indivíduos que podem ou não ter sido tratados por certo programa. Seja $D_{i}=\{0,1\}$ uma variável binária que marca se o indivíduo $i$ participou ou não do programa, com $D_{i}=1$ se participou, e $D_{i}=0$, caso contrário. Existem, portanto, $N_{1}=\sum_{i=1}^{N} D_{i}$ indivíduos tratados e $N_{0}=\sum_{i=1}^{N}\left(1-D_{i}\right)$ não tratados, de tal forma que $N_{1}+N_{0}=N$. Seja $X_{i}$ o vetor de $K$ variáveis que representam as características observáveis do indivíduo $i$ e $Y_{i}$ uma variável de resultado sobre a qual se tem interesse em estimar o efeito do programa.

Utilizando a noção de resultado potencial, podemos escrever: $Y_{i}=D_{i} Y_{i}^{1}+$ $\left(1-D_{i}\right) Y_{i}^{0}$, em que $Y_{i}^{1}$ representa a variável de resultado que o indivíduo $i$ teria no caso de ser tratado e $Y_{i}^{0}$ no caso de não passar pelo programa. Uma das vantagens dessa notação é que ela explicita que o resultado para um indivíduo tratado não necessariamente é igual ao que o mesmo indivíduo teria caso não passasse pelo tratamento. Na realidade, se o programa teve algum efeito, deve-se esperar que $Y_{i}^{1} \neq Y_{i}^{0}$. Essa notação também explicita que, como um indivíduo não pode estar nas duas situações simultaneamente, é necessário buscar uma forma de identificar $Y_{i}^{0}$ ou $Y_{i}^{1}$ (aquele que não for observado).

Estamos interessados em estimar o efeito do programa sobre os tratados. Esse efeito corresponde a um parâmetro que é definido pela diferença entre a média da variável de resultado para os tratados e a média da mesma variável para os tratados na situação contrafactual:

$$
\tau=E\left[Y_{i}^{1} \mid D_{i}=1\right]-E\left[Y_{i}^{0} \mid D_{i}=1\right] .
$$

O primeiro termo é diretamente observado nos dados e pode ser estimado de forma direta por meio de: $\bar{Y}^{1}=N_{1}^{-1} \sum_{i: D_{i}=1} Y_{i}$. Todavia, não é possível observar o segundo termo e, assim, para identificá-lo é necessário recorrer a algumas hipóteses.

Em seguida passamos a uma exposição detalhada de cada um dos métodos aqui empregados. Antes, porém, destacamos o fato de que nossas estimativas do efeito do programa Q são todas relativas ao programa de aprendizes. Isso significa que estimativas positivas (negativas) para empregabilidade não de- 
vem ser interpretadas como: participar do programa Q é melhor (pior) para a empregabilidade do jovem do que não participar, mas, sim, como: se qualificar pelo programa Q é melhor (pior) para a empregabilidade do jovem do que se qualificar pela aprendizagem. Em um caso extremo, pode acontecer de ambos os tipos de qualificação impactarem positivamente uma determinada dimensão avaliada, mas obtermos um sinal negativo devido a um maior impacto da aprendizagem.

\subsection{Pareamento com escore de propensão}

Neste estudo, empregamos o método de pareamento com escore de propensão que requer duas hipóteses básicas. A primeira, denominada hipótese de independência condicional, requer que a variável de resultado na ausência do tratamento seja independente da participação no programa condicionada às características observáveis dos indivíduos, ou seja:

$$
\text { (H1) } \quad Y_{i}^{0} \perp D_{i} \mid X_{i}
$$

Em outras palavras, essa hipótese sustenta que o vetor de variáveis em $X_{i}$ contém todas as informações que permitem considerar a decisão de participar ou não do programa, $D_{i}$, independente do resultado que o indivíduo teria na ausência do mesmo, $Y_{i}^{0}$. Na realidade, para os nossos propósitos, precisamos apenas de uma versão especializada dessa hipótese que requer que, ao controlar por $X_{i}$, a média de $Y_{i}^{0}$ seja independente da participação ou não no programa, $D_{i}$ :

$$
\text { (EH1) } \quad E\left[Y_{i}^{0} \mid D_{i}, X_{i}\right]=E\left[Y_{i}^{0} \mid X_{i}\right] .
$$

A principal implicação de H1 (ou EH1) é que indivíduos não tratados com características observáveis iguais às dos indivíduos do grupo tratado fornecem uma base para se obter o contrafactual desejado. Especificamente, supõe-se que os resultados de não tratados com o mesmo $X$ que o de tratados representem bem o que seria o resultado dos últimos na ausência do programa.

Naturalmente, para atender H1 (ou EH1) é necessário encontrar indivíduos não tratados com as mesmas características dos indivíduos tratados. No entanto, se o vetor $X_{i}$ tiver dimensão elevada, será muito difícil atender esse requerimento. A solução típica para esse problema de dimensionalidade é empregar o escore de propensão, que é uma função que condensa as informações contidas no vetor $X_{i}$. Ele é definido por:

$$
p\left(X_{i}\right) \equiv P\left[D_{i}=1 \mid X_{i}\right] .
$$

Mas, mesmo com o uso do escore de propensão, é preciso garantir que as características dos indivíduos tratados sejam representadas no grupo dos não tratados. Assim, a segunda hipótese requerida pelo método de pareamento com escore de propensão é que haja sobreposição nos escores de propensão entre tratados e não tratados. Formalmente, essa hipótese, conhecida como condição de suporte comum, requer que: ${ }^{5}$

\footnotetext{
${ }^{5} \mathrm{Na}$ realidade, para identificar o efeito do tratamento sobre os tratados, precisamos apenas que $p\left(X_{i}\right)<1$.
} 


$$
0<p\left(X_{i}\right)<1 .
$$

Implicitamente, $\mathrm{H} 2$ exige a existência de valores de $X$ válidos tanto para os tratados quanto para os não tratados. Caso ela seja violada, não há garantias de que isso será respeitado.

Sob as hipóteses H1 e H2, podemos então identificar o efeito do programa sobre os tratados com características $X_{i}=x$. Esse efeito pode ser escrito como:

$$
\tau(x)=E\left[Y_{i}^{1} \mid D_{i}=1, X_{i}=x\right]-E\left[Y_{i}^{0} \mid D_{i}=1, X_{i}=x\right] .
$$

Para identificá-lo, precisamos apenas encontrar uma maneira de computar o segundo termo, já que o primeiro pode ser estimado diretamente dos dados com base em indivíduos tratados com a combinação de características $X_{i}=x$. Usando a hipótese EH1, o segundo termo de (3) se transforma em:

$$
\begin{aligned}
& E\left[Y_{i}^{0} \mid D_{i}=1, X_{i}=x\right]=E\left[Y_{i}^{0} \mid D_{i}=0, X_{i}=x\right] \\
& \quad=E\left[Y_{i} \mid D_{i}=0, X_{i}=x\right]
\end{aligned}
$$

o que mostra que a média da variável de resultado dos tratados com características $X_{i}=x$ na ausência do programa é identificada pela média correspondente dos não tratados com a mesma combinação de características. A conjugação da hipótese de sobreposição $\mathrm{H} 2$ com esse resultado garante que a média $E\left[Y_{i}^{0} \mid D_{i}=1, X_{i}=x\right]$ pode ser identificada com base nos indivíduos pertencentes ao grupo de não tratados, que pode ser denominado grupo de controle.

Para obtermos o efeito geral do programa sobre os tratados, basta calcular a média de $\tau(x)$ na distribuição de $X$ para os tratados:

$$
\begin{aligned}
\tau & =E_{X \mid D_{i}=1}[\tau(x)]=E_{X \mid D_{i}=1}\left[E\left[Y_{i}^{1} \mid D_{i}=1, X_{i}=x\right]\right. \\
& \left.-E\left[Y_{i} \mid D_{i}=0, X_{i}=x\right]\right]
\end{aligned}
$$

nos quais fizemos uso da expressão (4) na segunda igualdade. Expressão análoga a essa pode ser escrita condicionando em $p\left(X_{i}\right)$ em vez de condicionar em $X_{i}$.

Neste estudo, o pareamento entre tratados e controles foi realizado com base no uso do escore de propensão. ${ }^{6}$ Como a forma funcional desse escore não é conhecida, empregamos um procedimento paramétrico amplamente usado na literatura para estimá-lo, especificamente o modelo Logit, dado por: $p\left(X_{i}\right)=P\left[D_{i}=1 \mid X_{i}\right]=\frac{\exp \left(X_{i}^{\prime} \theta\right)}{1+\exp \left(X_{i}^{\prime} \theta\right)}$, em que $\theta$ é um vetor de parâmetros. O valor predito do escore de propensão, $\hat{p}\left(X_{i}\right)=\frac{\exp \left(X_{i}^{\prime} \hat{\theta}\right)}{1+\exp \left(X_{i}^{\prime} \hat{\theta}\right)}$, foi obtido para todos os indivíduos da amostra, tratados e controles, e a condição de suporte

\footnotetext{
${ }^{6}$ Rosenbaum \& Rubin (1983) mostram que, se $\mathrm{H} 1$ for válida, então $Y_{i}^{0} \perp D_{i} \mid p\left(X_{i}\right)$ também é. Analogamente, se EH1 for verdadeira, então $E\left[Y_{i}^{0} \mid D_{i}, p\left(X_{i}\right)\right]=E\left[Y_{i}^{0} \mid p\left(X_{i}\right)\right]$.
} 
comum foi aplicada. ${ }^{7}$ Para parear os indivíduos tratados com os do grupo de controle, empregamos o procedimento de raio (radius), que utiliza para cada indivíduo tratado $i$ um subconjunto de indivíduos do grupo de controle cujos escores de propensão preditos se encontram a uma distância preestabelecida (isto é, o raio) do escore de propensão predito do indivíduo $i .^{8}$ O cômputo da média da variável de resultado contrafactual para cada indivíduo tratado $i$ foi obtida então usando a variável de resultado desse subconjunto de indivíduos do grupo de controle. O efeito global do programa sobre os tratados utiliza a média dessas médias calculada para o conjunto de todos os tratados (no suporte comum) e a média da variável de resultado para os próprios tratados. ${ }^{9}$

\subsection{Estimador de regressão linear de Oaxaca-Blinder}

É possível especializar o método de pareamento para um no qual se assume que a equação que governa o resultado contrafactual dos tratados tem formato linear. Especificamente, assume-se que:

$$
Y_{i}^{0}=X_{i}^{\prime} \beta^{0}+\varepsilon_{i}^{0}
$$

onde $\beta^{0}$ é um vetor de parâmetros (incluindo o intercepto) e $\varepsilon_{i}^{0}$ um termo de distúrbio aleatório. $\mathrm{O}$ equivalente da hipótese de independência condicional, EH1, aplicada nesse contexto linear é:

$$
\text { (LEH1) } \quad E\left[\varepsilon_{i}^{0} \mid X_{i}, D_{i}\right]=0 .
$$

Essa hipótese pode ser interpretada como uma exigência de que o controle pelas características observáveis em $X$ torna, em média, o distúrbio aleatório, $\varepsilon_{i}^{0}$, independente da decisão de participar ou não do programa, $D_{i}$. Assumindo sua validade, é possível estimar a média da variável de resultado contrafactual para os tratados com base somente na amostra de indivíduos não tratados. Por (6) e (LEH1), temos que:

$$
\begin{gathered}
\mu_{0}^{1}=E\left[Y_{i}^{0} \mid D_{i}=1\right]=E\left[X_{i} \mid D_{i}=1\right]^{\prime} \beta^{0}=E\left[X_{i} \mid D_{i}=1\right]^{\prime} \\
\left\{E\left[X_{i} X_{i}^{\prime} \mid D_{i}=0\right]^{-1} E\left[X_{i} Y_{i} \mid D_{i}=0\right]\right\}
\end{gathered}
$$

em que a última igualdade usa o fato de que $\beta^{0}$ pode ser obtido pela regressão

\footnotetext{
${ }^{7}$ Para o caso de emprego no mesmo ano ou ano seguinte ao treinamento, o valor mínimo do escore de propensão foi 0 e o valor máximo foi 0,937 . Para o caso de emprego dois ou três anos após o treinamento, os valores foram 0 e 0,959 .

${ }^{8}$ Utilizamos também os métodos de "vizinho mais próximo" e de Kernel, e os resultados foram bastante próximos dos obtidos com o método do raio.

${ }^{9}$ Formalmente, se $\left|\hat{p}\left(X_{j}\right)-\hat{p}\left(X_{i}\right)\right|$ é a distância entre os escores preditos dos indivíduos $\mathbf{j} \neq \mathbf{i}$, o conjunto de indivíduos do grupo de controle cujos escores preditos se situam a distância $\mathrm{r}$ do escore predito do indivíduo tratado $i$ é formado por $l(i \mid r)=\left\{j:\left|\hat{p}\left(X_{j}\right)-\hat{p}\left(X_{i}\right)\right| \leq r\right\}$. Assim, a média do contrafactual para $i$ é dada por $\widehat{Y}_{i}^{0}=N_{i}^{-1} \sum_{j \in l_{R}(r \mid i)} Y_{j}$, em que $N_{i}$ é o número de indivíduos do conjunto $l(i \mid r)$. O efeito do programa sobre os tratados é obtido então por: $\hat{\tau}=$ $\frac{1}{N_{1}} \sum_{i: D_{i}=1}\left(Y_{i}^{1}-\widehat{Y_{i}^{0}}\right)$.
} 
$Y$ em $X$ apenas para os não tratados. Na prática, denotando por $\beta^{0}$ o vetor de coeficientes estimados nessa regressão, a expressão (7) torna-se:

$$
\hat{\mu}_{0}^{1}=\bar{X}_{1}^{\prime} \hat{\beta}^{0}
$$

em que $X_{1}$ é a média das variáveis observáveis para o grupo de tratados. O efeito do programa sobre os tratados pode ser então estimado por:

$$
\hat{\tau}={\hat{\mu_{1}}}^{1}-{\hat{\mu_{0}}}^{1},
$$

em que $\bar{\mu}_{1}^{1}=\bar{Y}^{1}=N_{1}^{-1} \sum_{i: D_{i}=1} Y_{i}$ é a média do resultado calculada diretamente com base na amostra de tratados. ${ }^{10}$

Apesar de simples, o método da regressão linear depende das hipóteses de que a média condicional da variável de resultado é linear e de que as médias das variáveis em $X$ para tratados e não tratados são similares. ${ }^{11}$ Kline (2011) mostra, no entanto, que se o modelo linear para média condicional for correto ou se a probabilidade condicional de participação no programa (i.e., o escore de propensão) for linear, o estimador de regressão do efeito do programa sobre os tratados será não enviesado. ${ }^{12}$ Em particular, o autor mostra que, se o modelo para média condicional for linear, o método de regressão identifica esse efeito mesmo se a condição de suporte comum não for válida e/ou o modelo para o escore de propensão estiver incorreto. O autor também chama a atenção para a vantagem desse método no contexto em que se tem relativamente poucas observações tratadas e muitas no grupo de controle. Nossos dados se enquadram nesse contexto.

\section{Resultados}

Conforme mencionado na seção anterior, avaliaremos cada dimensão de desempenho no mercado de trabalho usando dois métodos distintos. Ao computar os estimadores, refinamos ainda mais nossa amostra introduzindo mais dois filtros em relação aos que foram listados na seção 3.1. Em primeiro lugar, limitamos o tamanho mínimo do estabelecimento de treinamento para 200 empregados, tendo em vista a diferença reportada na Tabela 2 para esse indicador entre os grupos de participantes dos programas $Q$ e de aprendizagem. Além disso, excluímos do grupo qualificado pelo programa $\mathrm{Q}$ aqueles jovens que se empregam como aprendizes após o término do programa $\mathrm{Q}$.

\footnotetext{
${ }^{10} \mathrm{O}$ desenvolvimento desse tipo de estimação tem como marco os trabalhos seminais de Oaxaca (1973) e Blinder (1973), motivando o uso da expressão Oaxaca-Blinder para estimadores desse tipo.

${ }^{11}$ É possível mostrar que $\hat{\mu}_{0}^{1}=\bar{X}_{1}^{\prime} \hat{\beta}^{0}=\bar{Y}^{0}+\left(\bar{X}_{1}-\bar{X}_{0}\right)^{\prime} \hat{\beta}^{0}$, em que $Y^{0}$ e $\dot{X}_{0}$ correspondem às médias da variável de resultado e das covariáveis para o grupo de controle, respectivamente. Essa expressão revela que, sob a hipótese LEH1, a estimação da média contrafactual desejada conterá viés sempre que $\bar{X}_{1}$ for diferente de $\bar{X}_{0}$.

${ }^{12}$ Note-se que basta que um dos dois condicionantes seja verdadeiro. Essa é uma característica dos estimadores denominados "duplamente robustos" (Robins et al. 1994).
} 


\subsection{Resultados para o curto prazo}

A Tabela 5 reporta nossas estimativas para diferentes dimensões do desempenho dos jovens no mercado de trabalho formal no curto prazo (i.e., até o ano seguinte ao término da qualificação). Na primeira coluna aparecem os resultados pelo método semiparamétrico de pareamento com escore de propensão, e na segunda, o resultado análogo obtido pelo método paramétrico de regressão linear baseado em Oaxaca-Blinder.

Na primeira linha da Tabela 5, reportamos as estimativas do efeito do programa $\mathrm{Q}$ relativamente à aprendizagem sobre a probabilidade de os jovens encontrarem qualquer tipo de emprego formal no curto prazo. Embora as estimativas pontuais obtidas pelos dois métodos sejam positivas, somente a do método linear é estatisticamente significativa e, mesmo assim, apenas ao nível de significância de $10 \%$. Esses resultados indicam que não há um impacto diferenciado sobre a empregabilidade geral dos jovens participantes do programa Q relativamente aos aprendizes no setor formal.

Nas linhas seguintes da Tabela 5, procuramos qualificar o tipo de emprego formal obtido, estimando em que medida o programa $Q$ impacta os jovens para alcançarem empregos de melhor qualidade. Por ser de difícil mensuração, adotamos diferentes alternativas para a noção de qualidade do emprego.

Em primeiro lugar, olhamos para a dimensão da estabilidade do vínculo e, para tanto, computamos os efeitos sobre a probabilidade de os jovens obterem empregos regidos por contratos por prazo indeterminado no horizonte de tempo de até um ano após o fim da qualificação. Novamente, não podemos dizer que há um efeito diferenciado do programa $Q$ em relação à aprendizagem, visto que as estimativas por ambos os métodos aqui empregados não são significativas do ponto de vista estatístico.

Uma vez que empregos de mais longa duração tendem a aumentar a produtividade do trabalho e o salário, outro indicador da qualidade do emprego é a permanência do trabalhador na mesma empresa. Esse indicador é particularmente relevante para jovens, uma vez que eles têm pouco histórico no mercado de trabalho e, portanto, o período de treinamento permite que o empregador conheça melhor sua capacidade de trabalho, mitigando assim os problemas típicos de assimetria informacional. Ademais, o conhecimento específico adquirido pelo trabalhador e o fato de o treinamento na mesma empresa envolver investimento por parte do empregador deveriam aumentar as chances de o jovem permanecer empregado no mesmo estabelecimento. Os resultados na terceira linha da Tabela 5 apontam para um impacto relativo desfavorável ao programa $Q$, já que o jovem qualificado por esse programa apresenta uma menor probabilidade de permanecer no mesmo estabelecimento que o jovem qualificado pela aprendizagem. O primeiro (segundo) método aponta para uma diferença na probabilidade de permanência de $6,2(3,7)$ pontos de porcentagem entre jovens aprendizes e os oriundos do programa Q. Essas estimativas são estatisticamente significativas e de magnitude elevada, pois a média dos jovens dos dois grupos que permanecem na mesma empresa após o período de aprendizado é de aproximadamente $19,4 \%$ (16,2\% no caso de aprendizes e $25,5 \%$ no caso do programa Q).

Outra maneira de acessarmos a qualidade do posto de trabalho eventualmente alcançada pelo jovem recém-qualificado é utilizar características da empresa usualmente associadas a postos de boa qualidade. Empregamos aqui duas medidas nessa direção: a probabilidade de os jovens se empregarem após 
Tabela 5: Resultados para emprego até um ano após o fim do treinamento, pelos métodos de pareamento (escore de propensão) e Oaxaca-Blinder

\begin{tabular}{lcc}
\hline Variáveis & Pareamento & Oaxaca-Blinder \\
\hline Emprego com qualquer tipo de contrato & 0,002 & $0,027^{*}$ \\
& $(0,017)$ & $(0,015)$ \\
Emprego com contrato por tempo indeterminado & $-0,016$ & 0,012 \\
& $(0,018)$ & $(0,015)$ \\
Emprego com qualquer tipo de contrato na mesma & $-0,062^{* * *}$ & $-0,037^{* * *}$ \\
empresa do treinamento & $(0,013)$ & $(0,014)$ \\
Emprego com qualquer tipo de contrato em empresa & $0,208^{* * *}$ & $0,204^{* * *}$ \\
com mais de 200 funcionários & $(0,014)$ & $(0,012)$ \\
Emprego com qualquer tipo de contrato em empresa & $0,188^{* * *}$ & $0,196^{* * *}$ \\
acima do p75 de salário médio & $(0,017)$ & $(0,015)$ \\
Log da remuneração média por hora de trabalho & $-0,019$ & 0,015 \\
no emprego & $(0,021)$ & $(0,014)$ \\
\hline
\end{tabular}

Fonte: Rais.

Elaboração dos autores.

Obs.: 1. Níveis de significância: ${ }^{*} 10 \%,{ }^{* *} 5 \%,{ }^{* * *} 1 \%$. Erros-padrão entre parênteses.

2. Na primeira coluna, utilizamos o método de pareamento por radius, em que cada indivíduo tratado é pareado com todos os indivíduos do grupo de controle cujos escores de propensão estejam em um raio $(0,1)$ ao redor do valor calculado para aquele tratado. Na segunda coluna, utilizamos o método proposto por Kline (2011) e descrito em detalhes na seção 4.3. Os controles utilizados em ambos os métodos foram: dummy de experiência anterior ao treinamento, faixa de tamanho da empresa (200-500 ou 500+ funcionários), dummies indicando se a empresa está situada na capital ou na região metropolitana das respectivas capitais, interação linear entre ano de treinamento e UF e entre ano de treinamento e faixa salarial da empresa (salário médio pago pela empresa), ano de treinamento, UF da empresa, setor da empresa, faixa de escolaridade e faixa salarial da empresa. Todas as variáveis relacionadas às empresas são referentes às empresas de treinamento, assim como as variáveis individuais consideram valores do último ano de treinamento do indivíduo.

a qualificação em empresas com mais de 200 funcionários (quarta linha da Tabela 5) ou em empresas que pagam salários relativamente altos, aqui demarcadas como as empresas que estavam entre as $25 \%$ com salários médios mais altos (quinta linha). Os resultados são positivos, estatisticamente significativos, com magnitudes elevadas e parecidas em ambos os métodos. Os jovens qualificados no programa Q têm cerca de 20,8 pontos de porcentagem a mais de chance de serem empregados em empresa com mais de 200 funcionários após a conclusão da qualificação do que os jovens qualificados pela aprendizagem. No que diz respeito a empregos em empresas que pagam salários relativamente altos, os jovens qualificados do programa $Q$ possuem uma probabilidade em torno de 18,8 pontos de porcentagem mais alta de se empregarem em empresas desse tipo do que os jovens qualificados pela aprendizagem.

Finalmente também analisamos o logaritmo do salário eventualmente obtido pelo jovem no posto de trabalho após a qualificação. Nessa dimensão de qualidade, temos novamente uma situação na qual não é possível afirmar que há uma distinção do ponto de vista estatístico do resultado alcançado pelo grupo que se qualificou pelo programa $Q$ ou pela aprendizagem. Vale notar que usamos o salário padronizado por horas trabalhadas.

Em suma, o jovem qualificado pelo programa $Q$ parece ter as mesmas chances de encontrar um emprego do que os jovens qualificados pela aprendizagem, em um horizonte de tempo que vai desde a conclusão da qualificação até 
o ano seguinte. Também parece não haver distinção no salário entre os jovens dos dois grupos que se empregam no horizonte considerado. Quando olhamos para a qualidade do emprego, o programa $\mathrm{Q}$ se mostra efetivo para que o jovem consiga emprego em empresas maiores e que pagam relativamente mais (maior salário médio). Porém, quando consideramos a permanência do jovem na mesma empresa, o resultado se torna favorável ao aprendiz, que tem maior probabilidade de ser efetivado na empresa de treinamento. Esse é um resultado intrigante, que merece uma atenção especial.

\subsection{Interpretação do resultado}

A menor probabilidade de permanência na mesma empresa do jovem treinado pelo programa $Q$, relativa à do jovem aprendiz, poderia estar associada a um maior número de treinandos relativo ao número de vagas disponíveis nas empresas que aderem ao programa $\mathrm{Q}$ do que nas empresas que aderem à aprendizagem. Essa hipótese é difícil de ser verificada diretamente, dado que não dispomos do número de vagas disponíveis nas empresas. Assumindo que o número de vagas é proporcional ao número de funcionários, podemos testar essa hipótese comparando a razão entre treinandos e o total de funcionários para os conjuntos de empresas que aderem a cada um dos tipos de treinamento. Essas razões estão reportadas na Figura 1.

Figura 1: Proporções entre a quantidade de treinandos em ambos os programas e a quantidade de funcionários das empresas de treinamento

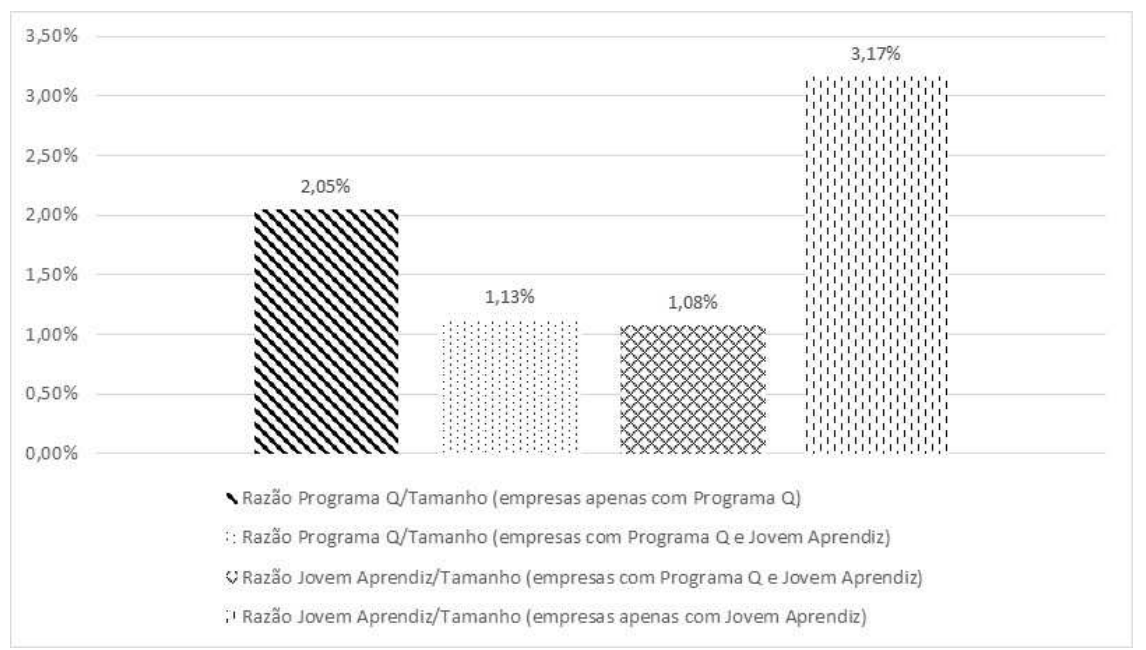

Fonte: Rais. Elaboração dos autores.

Nas duas primeiras barras, temos as razões em empresas que aderem ao programa Q. A primeira barra mostra a razão para o subconjunto de empresas que aderem ao programa $\mathrm{Q}$ mas não aderem à aprendizagem. Na segunda, temos a razão de treinandos no programa $\mathrm{Q}$ e o total de empregados das empresas que usam os dois tipos de treinamento. A terceira barra também se refere ao mesmo universo, porém, reporta à razão entre aprendizes e ao total de funcionários (excluindo aprendizes). Por fim, a quarta barra reporta à mesma razão da terceira para o universo de empresas que aderem à aprendizagem, mas não ao programa Q. Os resultados apontam que, nas empresas que 
possuem ambos os programas (representadas na segunda e na terceira barras), a proporção dos treinandos de ambos os programas é similar. Já nas empresas que apresentam apenas um dos programas, a quantidade relativa de aprendizes (quarta barra) é por volta de 50\% superior à dos treinandos do programa $\mathrm{Q}$ (primeira barra).

As evidências citadas não dão suporte à hipótese de que a relativa menor permanência do jovem treinado pelo programa $Q$ na mesma empresa poderia se dever a uma maior concorrência para esse jovem. Na verdade, a quantidade de aprendizes em relação ao tamanho da empresa é maior do que a verificada no programa $\mathrm{Q}$, indicando que haveria um maior número relativo de aprendizes concorrendo a cada vaga na empresa onde realizou o treinamento em comparação ao caso dos jovens do programa Q.

No entanto, ao aproximar o número de vagas pelo total de funcionários, estamos considerando que tanto o jovem treinado no programa $Q$, como o jovem treinado na aprendizagem estariam sendo considerados potenciais candidatos a um mesmo número de vagas (a rigor para quaisquer postos de trabalho da empresa que forneceu o treinamento). Sendo o conteúdo do treinamento do programa Q mais específico do que o da aprendizagem, é razoável supor que o conjunto de postos de trabalho onde esse jovem pode vir a ser aproveitado na empresa seja mais reduzido do que o disponível para o jovem aprendiz. Logo, não descartamos a hipótese de que a menor permanência do jovem treinado pelo programa Q na mesma empresa poderia se dever a uma maior concorrência enfrentada por esse jovem para um conjunto restrito de postos de trabalho.

\subsection{Resultados para o médio prazo}

Como para vários outros programas de treinamento, os impactos podem variar de acordo com o horizonte de tempo considerado. A literatura especializada em avaliação de programas de treinamento aponta que não é raro um resultado de impacto nulo no curto prazo, mas que aparece como positivo em um prazo mais extenso (Card et al. 2010, Kluve 2010). Uma das vantagens de utilizar a Rais para analisar efeitos de programas de treinamento (ou de outro tipo) é que os indivíduos podem ser seguidos por períodos mais longos. Aproveitando essa vantagem, implementamos também a avaliação do programa $Q$ para um horizonte de médio prazo, demarcado entre o início do segundo ano e o término do terceiro, após a conclusão da qualificação. Os resultados estão reportados na Tabela 6, que possui a mesma estrutura da Tabela 5.

Em geral, os resultados da Tabela 6 não diferem do ponto de vista qualitativo daqueles reportados na Tabela 5 para um horizonte mais próximo da conclusão da qualificação. Observam-se exceções para dois indicadores. O primeiro é que os resultados de emprego na mesma firma do treinamento deixam de ser distintos do ponto de vista estatístico entre os jovens do programa $\mathrm{Q}$ e os aprendizes. O segundo é que os resultados para remuneração passam a apontar uma distinção entre os dois grupos de jovens: o salário do jovem qualificado pelo programa Q no médio prazo tende a ser mais baixo do que o salário obtido pelo jovem qualificado pela aprendizagem. A magnitude dessa diferença é de 13,6\% no primeiro método, e de 15,5\% no segundo método, sendo ambos estatisticamente significativos. Essa diferença pode mais uma vez ser explicada pelo conteúdo mais específico do treinamento realizado inteiramente na empresa. Conforme argumentado em Biavaschi et al. (2013), esse fato pode fazer com que o jovem adquira menos "habilidades ocupacio- 
nais gerais" , ficando em uma posição de vulnerabilidade no mercado de trabalho. Isso porque para progredir no mercado de trabalho a tendência é passar por ocupações distintas que requerem diferentes habilidades ou formações. $\mathrm{O}$ teor muito específico do treinamento pode dificultar esse processo.

Tabela 6: Resultados para emprego de dois a três anos após o fim do treinamento, pelos métodos de pareamento (escore de propensão) e Oaxaca-Blinder

\begin{tabular}{lcc}
\hline Variáveis & Pareamento & Oaxaca-Blinder \\
\hline Emprego com qualquer tipo de contrato & 0,016 & 0,025 \\
& $(0,019)$ & $(0,017)$ \\
Emprego com contrato por tempo indeterminado & 0,021 & $0,034^{*}$ \\
& $(0,021)$ & $(0,018)$ \\
Emprego com qualquer tipo de contrato na mesma empresa & $-0,020$ & $-0,034$ \\
do treinamento & $(0,022)$ & $(0,023)$ \\
Emprego com qualquer tipo de contrato em empresa com mais & $0,273^{* * *}$ & $0,228^{* * *}$ \\
de 200 funcionários & $(0,027)$ & $(0,022)$ \\
Emprego com qualquer tipo de contrato em empresa acima do & $0,264^{* * *}$ & $0,220^{* * *}$ \\
p75 de salário médio & $(0,029)$ & $(0,024)$ \\
Log da remuneração média por hora de trabalho no emprego & $-0,136^{* * *}$ & $-0,155$ \\
& $(0,030)$ & $(0,021)$ \\
\hline
\end{tabular}

Fonte: Rais.

Elaboração dos autores.

Obs.: 1. Níveis de significância: * $10 \%,{ }^{* *} 5 \%,{ }^{* * *} 1 \%$. Erros-padrão entre parênteses.

2. Ver notas da Tabela 5 para detalhes de variáveis de controle e métodos.

\section{Conclusão}

Neste trabalho, avaliamos um programa inovador de qualificação de jovens composto por um treinamento oferecido dentro das empresas participantes do programa e com conteúdo ministrado pelos próprios empregados das respectivas empresas. Realizamos essa avaliação comparando a inserção futura no mercado de trabalho dos jovens participantes desse programa com os participantes do Programa Jovem Aprendiz. A escolha dos aprendizes como grupo de controle se deve à intenção de homogeneizar os jovens dos dois grupos pelo menos no que diz respeito a uma dimensão tipicamente não observada por avaliadores, a saber, a motivação por fazer algum curso de treinamento. A identificação dos efeitos do programa de interesse foi baseada no método de seleção em observáveis, que foi implementado por meio dos procedimentos de pareamento com escore de propensão e regressão linear baseado na decomposição de Oaxaca-Blinder. Fazemo-nos valer da riqueza de informações dos dados da Rais para considerar uma série de variáveis observáveis que procuram tornar os dois grupos comparáveis.

Estudamos os efeitos do programa com duas métricas de resultados: a empregabilidade dos participantes e os salários nos empregos pós-treinamento. Como os dados da Rais permitem seguir os trabalhadores ao longo dos anos, os efeitos foram estimados tanto para o curto (até um ano após o treinamento) quanto para o médio prazo (entre o segundo e o terceiro anos após o treinamento). Os resultados indicam que o programa avaliado não afeta a empregabilidade formal de seus participantes relativamente aos aprendizes nem no curto nem no médio prazo. Embora fosse de se esperar que uma iniciativa com as características do programa avaliado fosse aumentar a chance de seus 
participantes permanecerem na mesma empresa onde realizaram a capacitação, os resultados mostram que isso não ocorreu, pelo menos no curto prazo. O programa, no entanto, gera efeito positivo tanto no curto quanto no médio prazo em uma dimensão da qualidade do emprego: os jovens treinados pelo programa de interesse têm mais chance de se empregar em empresas maiores ou que pagam maiores salários do que os jovens treinados pela aprendizagem. Por outro lado, no que diz respeito aos efeitos sobre a remuneração, os resultados não apontam diferença entre os grupos no curto prazo, porém, uma diferença favorável à aprendizagem no médio.

Associamos os impactos negativos do programa sobre a empregabilidade a um elemento em comum: o teor mais específico do treinamento do programa $\mathrm{Q}$ em relação ao teor do treinamento na aprendizagem. Por um lado, as oportunidades de emprego dos jovens passam a ser restritas a um menor número de vagas na empresa de treinamento. Além disso, por ter desenvolvido um conjunto mais restrito de habilidades, uma progressão que dependa de promoções pode ser mais difícil para o jovem treinado pelo programa $Q$.

\section{Referências Bibliográficas}

Biavaschi et al., C. (2013), 'Youth unemployment and vocational training', Foundations and Trends in Microeconomics 9(1-2), 1-157.

Blinder, A. S. (1973), 'Wage discrimination: reduced form and structural estimates', The Journal of Human Resources 8(4), 36-455.

Calero et al., C. (2017), 'Can arts-based interventions enhance labor market outcomes among youth? Evidence from a randomized trial in Rio de Janeiro', Labour Economics 45, 131-142.

Card, D., Kluve, J. \& Weber, A. (2010), 'Active labour market policy evaluations: a meta-analysis.', The Economic Journal 120(548), 452-477.

Clark, K. B. \& Summers, L. H. (1982), Wage discrimination: reduced form and structural estimates, in R. B. Freeman \& D. A. Wise, eds, 'The youth labor market problem: its nature, causes and consequences', Chicago: University of Chicago Press.

Corseuil, C. H. \& Botelho, R. U. (2014), Desafios à trajetória profissional dos jovens brasileiros, Rio de Janeiro: Ipea.

Corseuil, C. H., Foguel, M. \& Gonzaga, G. (2015), 'Apprenticeship as a stepping-stone to better jobs: evidence from Brazilian matched employer-employee data', Rio de Janeiro: PUC-Rio. Texto para Discussão, n. 651.

Corseuil et al., C. H. (2014), A rotatividade dos jovens no mercado de trabalho formal brasileiro, in C. H. Corseuil \& R. U. Botelho, eds, 'Desafios à trajetória profissional dos jovens brasileiros', Rio de Janeiro: Ipea.

Cruces, G., Ham, A. \& Violas, M. (2012), 'Scarring effects of youth unemployment and informality: evidence from Argentina and Brazil', . Mimeografado.

Eliason, M. \& Storrie, D. (2006), 'Lasting or Latent Scars? Swedish evidence on the long-term effects of job Displacement', Journal of Labor Economics $4(24), 831-856$. 
Flori, P. (2005), 'Desemprego de jovens no Brasil', Revista da ABET 5(1), 2960.

Freeman, R. \& Wise, D. (1982), The youth labor market problem: its nature, causes and consequences., Chicago: University of Chicago Press.

Fundação Itaú Social (2015), 'Levantamento de estudos e avalia,ses sobre voluntariado', São Paulo.

Gregg, P. \& Tominey, E. (2005), 'The wage scar from male youth unemployment', Labour Economics 12(4), 487-509.

Huber, M., Lechner, M. \& Wunsch, C. (2013), 'The performance of estimators based on propensity score', Journal of Econometrics 175(1), 1-21.

Kline, P. (2011), 'Oaxaca-Blinder as a reweighting estimator', American Economic Review 101(3), 532-537.

Kluve, J. (2010), 'The effectiveness of European active labor market programs', Labour Economics 17(6), 904-918.

Leighton, L. \& Mincer, J. (1982), Labor turnover and youth unemployment, in R. B. Freeman \& D. A. Wise, eds, 'The youth labor market problem: its nature, causes and consequences', Chicago: University of Chicago Press.

Ministério do Trabalho (Brasil, 2009), 'Manual da aprendizagem: o que é preciso saber para contratar o jovem aprendiz', Brasília: SPPE.

Oaxaca, R. (1973), 'Male-female wage differentials in urban labor markets', International Economic Review 14(3), 693-709.

OECD (2014), 'Investing in youth: Brazil', Paris: OECD Publishing.

Robins, J., Rotnitzky, A. \& Zhao, L. (1994), 'Estimation of regression coefficients when some regressors are not always observed', Journal of the American Statistical Association 89(427), 846-866.

Rosenbaum, P. \& Rubin, D. (1983), 'The central role of the propensity score in observational studies for causal effects', Biometrika 70(1), 41-55. 
